\title{
MS054.001
}

Microsymposium

\section{Structural studies of clinical resistomes}

Albert Marinus Berghuis ${ }^{1}$, Angelia Bassenden ${ }^{1}$

${ }^{1}$ Department Of Biochemistry, McGill University, Montreal, Canada

E-mail: albert.berghuis@mcgill.ca

Resistance to antibiotics can be achieved through a number of different strategies. For the ribosome targeting aminoglycoside antibiotics, such as kanamycin and amikacin, the clinically most frequently observed mechanism of resistance is enzymatic modification of the drug. More than a hundred different enzymes have been identified in pathogenic bacteria that confer resistance to aminoglycosides. The enzymes can be classified into three main groups: those that phosphorylate, those that acetylate and those that adenylate the drugs. As a single enzyme can often provide resistance to multiple aminoglycosides, and it is not uncommon that a single pathogen harbours several different aminoglycoside modifying enzymes, resistance to these antibiotics is rampant. While the situation is dire, it must be realized that some enzymes are far more frequently encountered in hospital settings than others, and thus clinically relevant resistance to specific aminoglycoside antibiotics is often only mediated by three to five unique enzymes. We have performed crystallographic studies of aminoglycoside modifying enzymes that confer clinically observed resistance to two of the most widely used aminoglycoside antibiotics: tobramycin and gentamicin. Structural analyses of the tobramycin and gentamicin clinical resistomes reveals surprising diversity in observed drug-target interactions. Combining these insights with information on drug-target nteractions with the intended target, the bacterial ribosome, can furthermore inform the development of nextgeneration aminoglycoside antibiotics.

[1] Bacot-Davis, V.R. et al (2016) Med. Chem. Commun. 7, 103-113.

[2] Bassenden, A.V. et al (2016) ACS Chem. Biol. 11, 1339-1346.

Keywords: aminoglycosides, drug-development, enzymes 